\title{
Deep Learning-Based Analysis of Efficiency and Surgical Timing for Patients with Cervical Insufficiency Using Transvaginal Ultrasound Images
}

\author{
Xuekui Ye $\mathbb{D}^{\mathbb{1}},{ }^{1}$ Li Zhang $\mathbb{D}^{1},{ }^{1}$ Rongxia Liu $\mathbb{D}^{1},{ }^{1}$ Yongjuan Liu $\mathbb{D}^{1},{ }^{1}$ and Guowei Jiang $\mathbb{D}^{2}$ \\ ${ }^{1}$ Department of Gynaecology, Shijiazhuang No. 4 Hospital, Shijiazhuang 050000, China \\ ${ }^{2}$ The Second Department of Obstetrics, Shijiazhuang No. 4 Hospital, Shijiazhuang 050000, China \\ Correspondence should be addressed to Guowei Jiang; 2017107146@ctgu.edu.cn
}

Received 22 September 2021; Revised 12 November 2021; Accepted 14 December 2021; Published 3 January 2022

Academic Editor: M Pallikonda Rajasekaran

Copyright (c) 2022 Xuekui Ye et al. This is an open access article distributed under the Creative Commons Attribution License, which permits unrestricted use, distribution, and reproduction in any medium, provided the original work is properly cited.

\begin{abstract}
Objective. This work aims to analyze the surgical timing and clinical efficacy of transvaginal cervical ring ligation based on the ultrasound image focus detection of patients with cervical insufficiency (CIC) under the ultrasound image theme generation model. Methods. 134 CIC patients who came to the hospital for ultrasound imaging diagnosis were collected. Observation group was treated with cervical cerclage (CC) and the pregnancy outcome was followed up. Control group was treated conservatively. Results. For patients in the control group, average gestational age was $21.12 \pm 2.18$ weeks, average cervical length (CL) was $15.54 \pm 0.42 \mathrm{~mm}$, and average uterine opening width was $3.06 \pm 0.63 \mathrm{~mm}$. In the observation group, average gestational age was $24.45 \pm 4.12$ weeks, average CL was $17.32 \pm 4.09 \mathrm{~mm}$, and average uterine opening width was $0.21 \mathrm{~mm}$. There were significant differences between the two groups $(P<0.05)$. There were also significant differences in the degree of uterine orifice dilation between the two groups $(P<0.05)$. Pregnancy outcomes of the two groups were compared, and $\chi^{2}$ and $P<0.05$ indicated significant differences. Conclusion. Convolution neural network (CNN) and long short-term memory model (LSTM) algorithm were used to classify patients' ultrasound images, which could effectively improve diagnosis and treatment efficiency. Surgical success rate, clinical outcomes, neonatal survival rate, and clinical effect of observation group were better than those of control group. Cervical ligation was best performed before 17 weeks of pregnancy in CIC.
\end{abstract}

\section{Introduction}

Patients with cervical insufficiency (CIC) is also called intra-cervix relaxation, usually refers to the miscarriage or premature birth due to anatomy or defects in the uterus without obvious contractions. According to research statistics, CIC is the main cause of recurrent miscarriage [1]. At present, the main clinical symptoms of the disease are habitual abortion and premature delivery caused by painless cervical expansion in the second or third trimester. The recurrence rate of the disease is as high as $2 \%$, which is about 3.3 times that of healthy women. It accounts for $8 \%-9 \%$ of all preterm birth data, and the highest rate of natural preterm birth can reach more than half [2]. Current medical experts and scholars have not clearly pointed out the pathogenesis of the disease, but it is classified into two types, structural insufficiency and functional insufficiency. Structural dysfunction is often referred to as congenital hypoplasia. Functional insufficiency usually refers to severe cervical damage caused by external effects, for example, the damage to the cervix during emergency labor, forceps, induced abortion, or curettage [3]. If the disease cannot be treated in time, it will recur and will have certain impacts on women's health and family [4]. CIC currently has no clear and unified diagnostic criteria internationally. There are only two main treatment methods: one is conservative expectant treatment, and the other is surgical treatment [5]. Conservative expectant treatment is mainly through bed rest, application of uterine contraction inhibitors, and antibiotics to fight infections [6]. Surgical treatment is mainly based on cervical ring ligation. Cervical ring ligation is 
currently an effective surgical method for the treatment of CIC, which can improve the perinatal outcome to a certain extent [7].

With the progression of modern technology, deep learning has penetrated all aspects of the computer vision field, and the analysis and application of medical images has become more and more extensive. Many experts and scholars have introduced deep learning into the field of ultrasound image understanding [8]. In the research and development in recent years, the adoption of deep learning has achieved good results in ultrasound images and retrograde classification, segmentation, and detection [9]. The topic generation technology of ultrasound images generates text information that describes the content of ultrasound images and the characteristics of the disease in the focus area through algorithms. Through the presentation of this information, inexperienced medical staff can directly use the generated text information to observe and understand the lesion information in the ultrasound image [10]. However, there are still some shortcomings in the analysis of content information in the current ultrasound image understanding. To automatically describe the supernatural image more quickly, the image theme generation technology was introduced in this work, to classify and interpret the information of the lesion in the ultrasound image [11] more quickly. First, convolution neural network (CNN) was employed to form a rough classification model to identify target organs in ultrasound images. Then, the long short-term memory model (LSTM) was employed to decode, and finally, the text information suitable for describing the content of the lesion area of the ultrasound image was acquired [12].

In summary, the topic generation technology based on deep learning ultrasound images is widely utilized in the field of clinical treatment, and it is also a hotspot of current research. Based on which, CNN and LSTM models were employed to understand and classify ultrasound images of CIC patients, to comprehensively evaluate the clinical application value of cervical ligation in the treatment of CIC patients.

\section{Materials and Methods}

2.1. Research Object. Patients who came to hospital from August 2019 to August 2020 and were diagnosed with CIC were deemed as the research objects. A total of 134 cases were enrolled, and they were between 20 and 40 years old. According to the random number table method, patients were divided into the observation group and the control group, with 67 patients in each group. In addition, 40 patients from the observation group were selected in group A, and the remaining 27 patients were selected in group $B$. The research had been approved by the ethics committee of the hospital. The patients and his family members were aware of the specifics of the study and had signed informed consent forms.

2.2. Inclusion Criteria. Inclusion criteria: (1) patient's gestational period reached 14-28 weeks, without vaginal bleeding, and surgery can be performed; (2) patient diagnosed as a CIC; (3) patients who were admitted to the hospital due to premature delivery or miscarriage, the uterine opening was more than $1 \mathrm{~cm}$ wide, and the amniotic fluid sac was visible in the uterine mouth.

Exclusion criteria: (1) the uterine mouth was too large, and abortion was inevitable; (2) reproductive system infection, urinary system infection, or active period of systemic infection, and obvious intrauterine infection before receiving fetal preservation treatment; (3) patients with incomplete clinical case data; (4) patients with serious medical and surgical complications and needed to stop pregnancy; (5) those who were lost to follow-up or research subjects withdrew halfway.

2.3. CNN and LSTM Construction. When processing some large images, the traditional network structure often faces three defects of the loss of information features, low computing efficiency, and network overfitting. CNN can deal with them better. The structure of $\mathrm{CNN}$ is basically composed of input layer, convolutional layer, ReLU layer, pooling layer, and full connection layer. The core network layer is the convolution layer, and most of the computation is carried out in the convolution layer. It carries on the filter learning, to recognize several specific types of image features will be activated. It is also regarded as an output of a neuron, and since convolution has the feature of "weight sharing" (Figure 1), the convolution layer can also reduce the number of parameters in the network structure and solve the overfitting of the network caused by many parameters.

There is usually a pooling layer between the adjacent convolutional layer and the convolutional layer. The pooling layer reduces the spatial size of data volume so that there will not be too many parameters in the network to reduce the operation efficiency and lead to overfitting phenomenon. There are several common functions that are applied to the pooling layer, but because maximum pooling works best, maximum pooling is often used in recent years. In practice, the maximum pooling layer is often presented as overlapping pooling and another larger receptive field pooling that destroys the network structure to some extent.

Linear rectification function (Rectified Linear Unit, ReLU), also known as modified linear unit, is a commonly used activation function in artificial neural networks. It usually refers to the nonlinear function represented by the ramp function and its variants, which is expressed as follows:

$$
f(x)=\max (0, x) .
$$

In $\mathrm{CNN}$, linear rectification is the activation function of the neuron, which defines the nonlinear output result of this neuron after linear transformation. In actual operation, the most suitable ReLU function should be selected as the activation function of the neuron. The fully connected layer (FC) is the last layer of the entire network structure of the CNN. It connects to other network layers in a fully connected way (Figure 2), which acts as a classifier in the entire $\mathrm{CNN}$. The operation of the convolutional layer, pooling layer, and activation function layer is mapping the original data to the hidden layer feature space, and the fully 


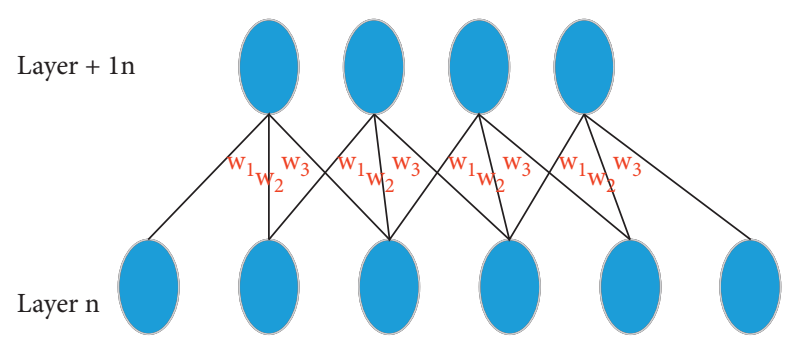

Figure 1: Weight sharing.

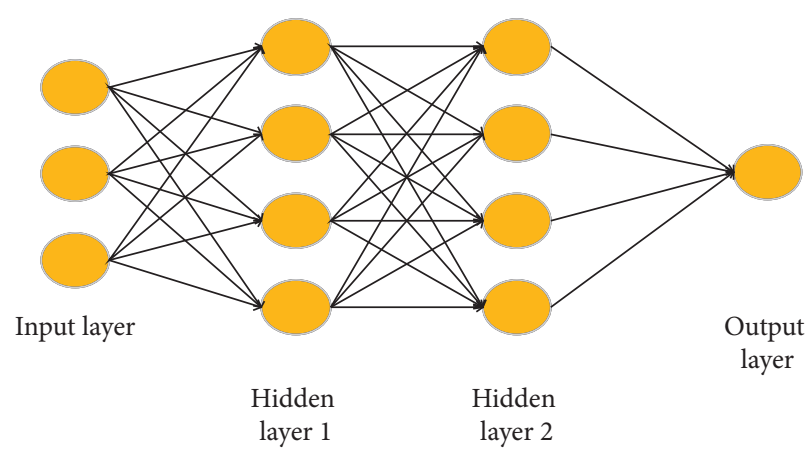

FIGURE 2: Fully connected layer.

connected layer maps the learned "distributed feature representation" to the sample label space.

Long short-term memory (LSTM) is a time loop neural network. Because recurrent neural network (RNN) generally has shortcomings such as long-term dependence, LSTM was developed to deal with this problem. LSTM contains LSTM blocks, which are usually regarded as a kind of intelligent network unit, which can memorize those unspecified values and then judge whether the input image is important enough to be memorized and then output.

There are mainly four core units in LSTM, namely, memory cell, forget gate, input gate, and output gate, as shown in Figure 3. One of the most important is the forget gate, followed by the input gate, and the last is the output gate. The three control units all contain two layers of the same fully connected network.

In LSTM, the input gate mainly involves two input methods: one is to manipulate the directly input data and the other is to input the data and store it in the memory unit for manipulation, as shown in equations (2) and (3), respectively:

$$
\begin{gathered}
\text { If }=\phi\left(\sum_{g} M_{f, g} d_{g}^{n}+\sum_{g} W_{f, g}, O_{g}^{(n-1)}+p_{f}\right), \\
I=\tanh \left(\sum_{g} M_{f, g} d_{g}^{n}+\sum_{g} W_{f, g}, O_{g}^{(n-1)}+p\right),
\end{gathered}
$$

where $O_{g}{ }^{(n)}$ represents the data input of $n, M_{f, g}$ represents the weight of the input, $W_{f}$, $g$ represents the weight of the loop (belonging to the forget gate), and $p_{f}$ represents the bias of the input gate. The forget gate maps the input data into the interval $[0,1]$ through sigmoid function. A value of 0 means that no message can pass through the control unit, while a value of 1 means that every message can pass through. The memory unit drill is repeated by the memory unit at each different moment, and when it needs to be adjusted, it is controlled by the input gate and the forgetting gate. The output gate works in the same way as the forget gate. When the value is 0 , it means that no information can pass through the control unit. When the value is 1 , it means that every information can pass through. The working principle of LSTM is the effective integration of important data through the loop memory of network structure and three control units.

2.4. Treatment Methods. The control group was treated conservatively, and ultrasound images were manually screened. According to the patient's pregnancy cycle and cervical condition, different uterine contraction inhibitors were given. Patients before 18 weeks of pregnancy were given intravenous injection of phloroglucinol. Ritodrine hydrochloride was adopted for patients at and after 20 weeks of pregnancy. Depending on the contractions, different doses were utilized. After 26 weeks of pregnancy, intravenous dexamethasone was given to promote lung maturity. After that, patients mainly rested in bed, minimized the time of getting out of bed, and follow-up was regularly made after surgery.

The observation group was treated with CC, and the ultrasound images were classified and compared by the CNN + LSTM model algorithm, manual sorting, and SDD300 algorithm.40 Forty patients in group $\mathrm{A}$ in the observation group received CC before 17 weeks of gestation. 


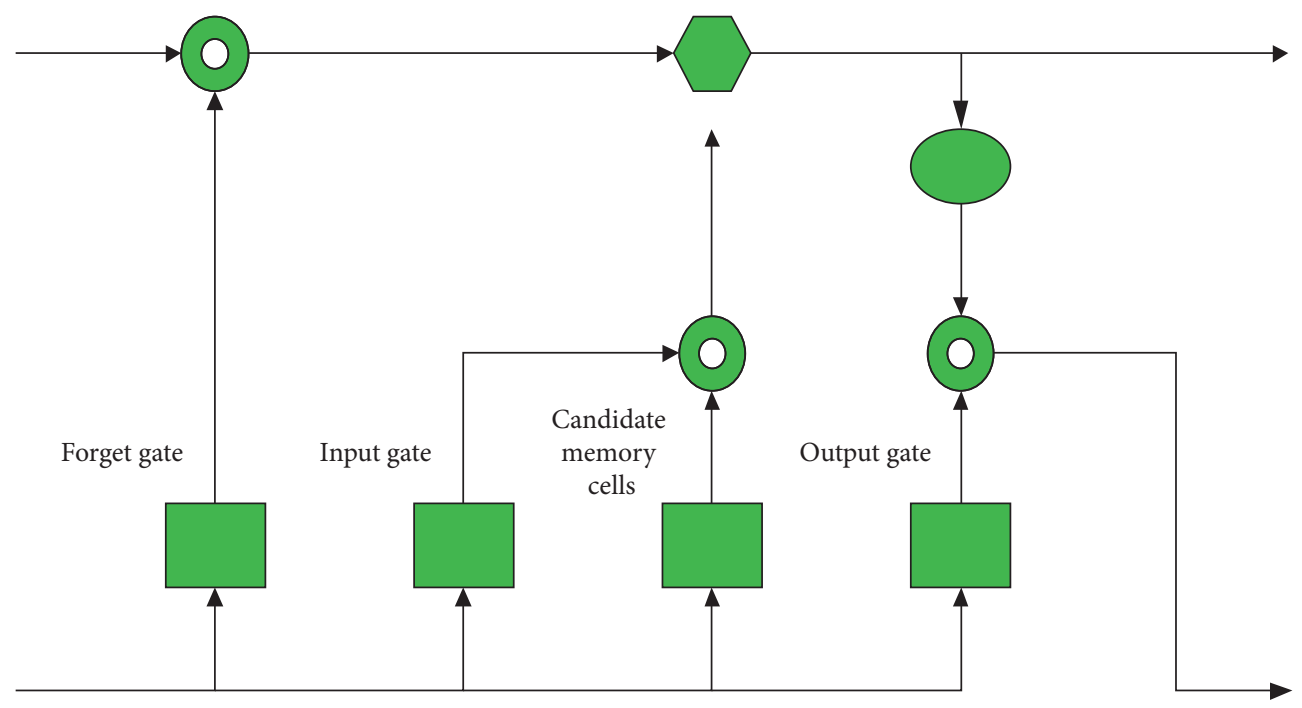

Figure 3: Basic model of LSTM network.

Twenty seven patients in group B underwent CC after 17 weeks of gestation. A series of routine tests were performed to detect contractions, and intravenous antibiotics were given before or during surgery to prevent infection. After the operation, conventional drugs were given to suppress contractions and patients should rest in bed. The vulva should keep clean, iodophor cleaning should be given if necessary, and follow-up was regularly made after surgery.

2.5. Observation Indicators. The general indicators of patients in the two groups were observed after admission and before treatment. The indicators such as age, height, weight, gestational age, number of pregnancies, number of births, $\mathrm{CL}$, and uterine opening width were recorded in detail. At the same time, patients were asked about their conception ways. After patients were treated, pregnancy outcomes were observed, and clinical effects of patients were analyzed in the two groups.

2.6. Statistical Analysis. The collected data were sorted by Excel and analyzed with SPSS22.0 statistical software. Mean \pm standard deviation $(\square x \pm s$ ) was how measurement data were expressed, and percentage (\%) was how count data were expressed. Pairwise comparison was implemented by the analysis of variance. $P<0.05$ indicated that the difference was statistically considerable.

\section{Results}

3.1. Comparison of Classification Results of Three Algorithms. Figure 4 shows the comparison of the sensitivity, specificity, accuracy, and running time of the three algorithms. The sensitivity of the SSD300 (Single Shot MultiBox Detector) algorithm was $90.12 \%$, the specificity was $88.32 \%$, the accuracy was $91.67 \%$, and the running time was $(15.28 \pm 6.82)$ $s$. The accuracy of manual sorting was $75.28 \%$, and the running time was $(80.73 \pm 10.28) s$. The sensitivity of the CNN + LSTM algorithm was $98.27 \%$, the specificity was

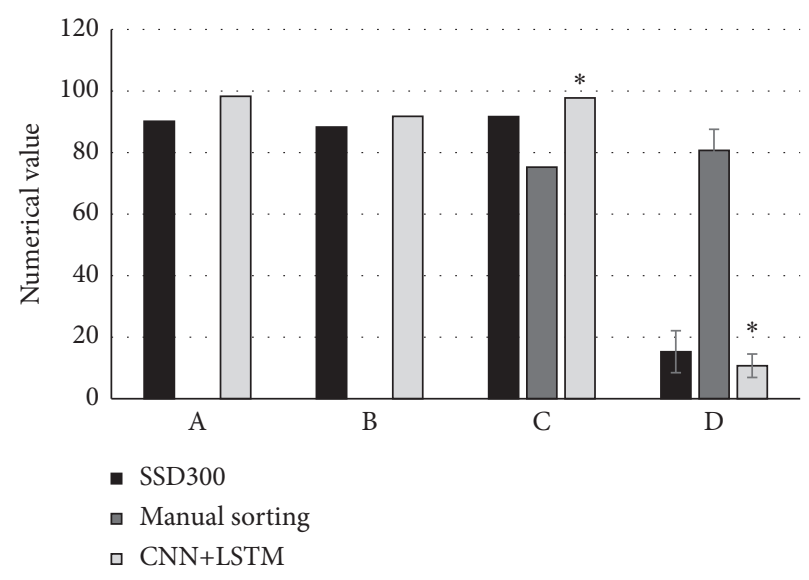

FIGURE 4: Contrast of three types of ultrasound image classification results: (a) sensitivity; (b) specificity; (c) accuracy; (d) running time ( ${ }^{*}$ meant $\left.P<0.05\right)$.

$91.73 \%$, the accuracy was $97.72 \%$, and the running time was $(10.72 \pm 3.81) s$. The sensitivity, specificity, and accuracy of the CNN + LSTM algorithm were obviously superior to those of SSD300 and manual sorting, and the differences were considerable $(P<0.05)$. The running time of CNN + LSTM was notably shorter than that of SSD300 and manual sorting $(P<0.05)$. Figures 5 and 6 show patients diagnosed as CIC by ultrasound image classification.

3.2. General Information of Patients. Among the collected 134 CIC patients, the average age of 67 patients in the observation group was $(31.5 \pm 3.78)$ years, the average weight was $(54.9 \pm 10.27) \mathrm{kg}$, and the average height was $(163.23 \pm 4.28) \mathrm{cm}$ (Figure 7$)$. The average number of pregnancies was $(3.02 \pm 1.11)$, the average number of births was $(0.78 \pm 0.49)$, and the average number of abortions was $(1.05 \pm 0.36)$ (Figure 8$)$. The average gestational age was $(24.45 \pm 4.12)$ weeks. The average cervical length at admission was $(17.32 \pm 4.09) \mathrm{mm}$, and the width of the internal 


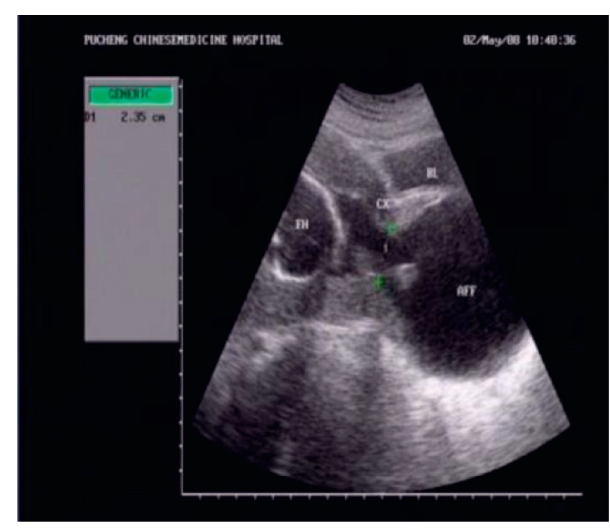

FIgURE 5: CIC image of a 27-year-old patient.

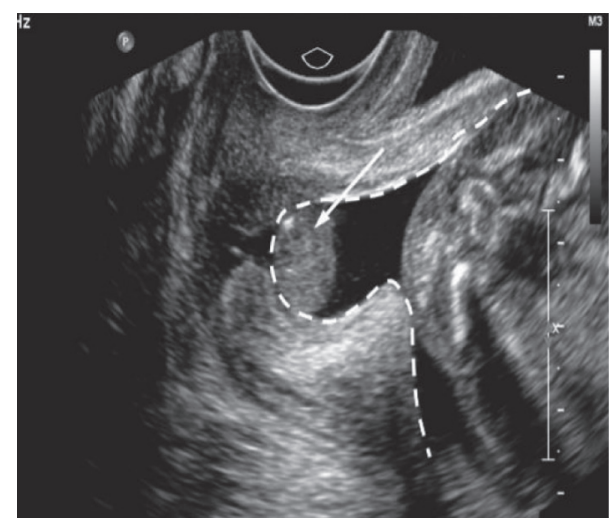

Figure 6: CIC image of a 31-year-old patient (the line indicated the cervix, and the arrow pointed to CIC.).

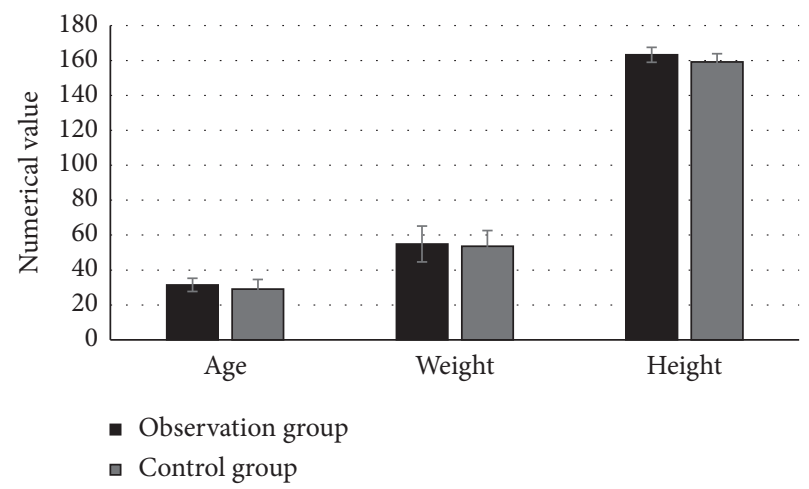

Figure 7: Basic indicators of the two groups of patients.

cervix at admission was $0.21 \mathrm{~mm}$ (Figure 9). The average age of 67 patients in the conservative treatment group was $(29.14 \pm 5.45)$ years, the average weight was $(53.63 \pm 8.98)$ $\mathrm{kg}$, and the average height was $(159.12 \pm 4.75) \mathrm{cm}$. The average number of pregnancies was $(2.89 \pm 1.77)$, the average number of births was $(0.11 \pm 0.47)$, and the average number of abortions was $(0.79 \pm 0.54)$. The average gestational week was $(21.12 \pm 2.18)$ weeks. The average length of the cervix at admission was $(15.54 \pm 0.42) \mathrm{mm}$, and the average width of the internal cervix at admission was $(3.06 \pm 0.63) \mathrm{mm}$.

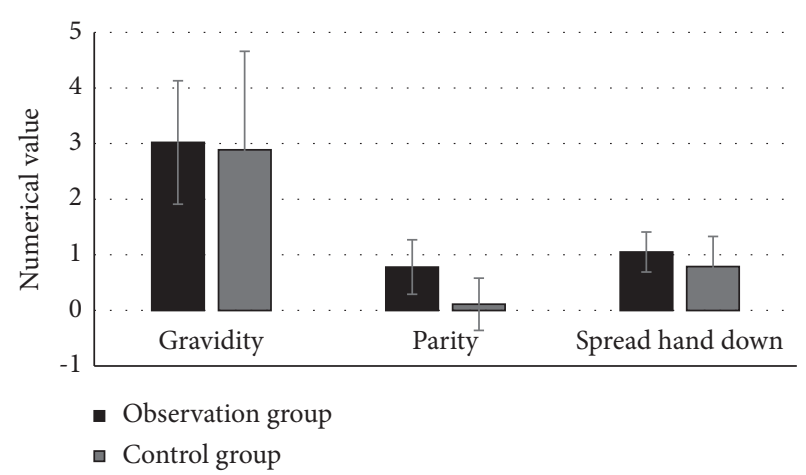

FIgUre 8: Pathological conditions of the two groups of patients.

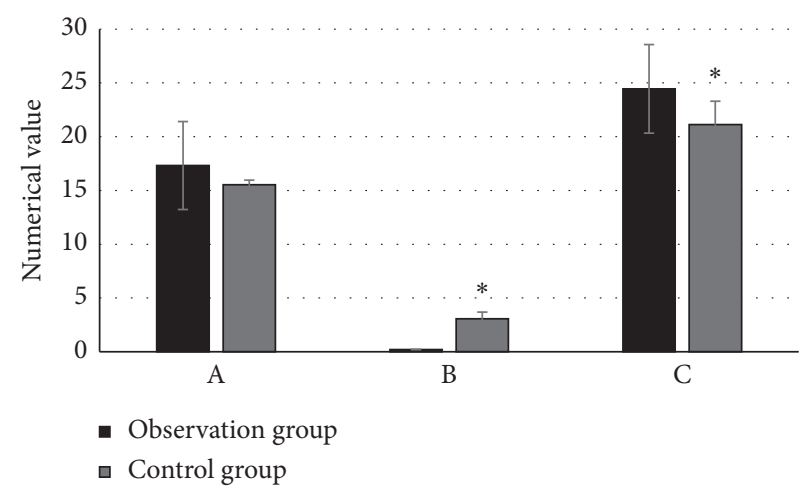

FIGURE 9: Contrast of cervical test results between the two groups. (a) Average CL of the patient at admission; (b) average cervical width of the patient at admission; (c) average pregnancy cycle of the patient.

Based on the above comparisons, the two groups of patients were not considerably different in age, pregnancy times, birth times, and number of abortions $(P>0.05)$. The original cervix length and the width of the internal cervix at the time of admission, as well as the pregnancy period of admission were compared, and those of the observation group was notably different from controls $(P<0.05)$.

3.3. Comparison of Conception Ways between the Two Groups. In the observation group, 47 cases were conceived naturally, accounting for $70.2 \%$, and 20 cases were conceived by assisted reproduction, accounting for $29.8 \%$. During treatment, the cervix was not opened in 66 cases, accounting for $98.5 \%$, and the cervix was opened in 1 case, accounting for $1.5 \%$. There were 50 cases of single pregnancy, 17 cases of twins, 7 cases of patients with a history of preterm birth, and 60 cases of patients without history of preterm birth. In the control group, 40 cases of natural conception, accounting for $60 \%$, and 27 cases of assisted reproduction conception, accounting for $40 \%$. During treatment, 59 patients had no cervical opening, accounting for $88 \%$, and 18 patients had cervical opening, accounting for $12 \%$. In the control group, 52 cases had single pregnancy and 15 cases had twin 
pregnancy. There were 5 patients with a history of preterm birth and 62 patients without a history of preterm birth. No considerable difference was indicated between groups of patients in terms of conception, number of fetuses, and history of preterm birth $(P>0.05)$. In the comparison of whether the uterine orifice was dilated, the observation group was obviously different from the control group $(P<0.05)$ (Figures 10 and 11).

3.4. Comparison of Pregnancy Outcomes between the Two Groups. Among the patients under investigation, in the observation group, 40 patients in group A underwent CC before 17 weeks, 36 cases were successfully treated with CC, the success rate was $89.6 \%, 4$ cases failed, and the failure rate was $10.4 \%$. In group B, 27 cases were treated with CC after 17 weeks. Nineteen cases were successfully treated with a success rate of $70.4 \%$, and 8 cases failed with a failure rate of 29.6\% (Figure 12). In the control group, 39 cases were successfully treated with conservative treatment, the operation success rate was $58.2 \%, 28$ cases failed, and the failure rate was $41.8 \%$ (Figure 13). Contrast of clinical effects between the two groups of patients showed that $\chi^{2}=27.539$, $P<0.000$, suggesting considerable difference. In summary, the observation group had a higher surgical success rate before the 17th week of gestation, and slightly lower surgical success rate after 17 weeks, but there was no remarkable difference between the two $(P<0.05)$. The observation group had a notably higher surgical success rate versus control group $(P<0.05)$.

In the observation group, 57 neonates survived successfully, accounting for $85.1 \%$, and 10 died, accounting for $14.9 \%$. Among controls, 40 neonates survived successfully, accounting for $59.7 \%$, and 27 died, accounting for $40.3 \%$ (Figure 14). Contrast of the neonatal survival rate of the two groups of patients suggested considerable differences between groups $(P<0.05)$.

\subsection{Comparison of Clinical Effects between the Two Groups.}

The clinical operation success rate of the two groups of patients was effective by prolonging 48 hours of fetal lung maturation treatment. In the observation group, 62 cases were treated with cervical ligation, the effective rate was $92.5 \%, 5$ cases were ineffective, and the failure rate was $7.5 \%$. Among controls, the number of effective cases of conservative treatment was 44 , the effective rate was $65.7 \%$, the number of ineffective cases was 23 , and the ineffective rate was $34.3 \%$ (Figure 15). Contrast of the length of prolonged pregnancy time between the two groups of patients suggested that $\chi^{2}=40.182, P<0.000$. The effective rate of observation group was remarkably superior to controls.

\section{Discussion}

CIC is caused by the failure of uterine anatomy or functional defects to maintain full-term pregnancy, and the incidence of late miscarriage is increasing day by day. In this work, CNN and LSTM model algorithms were constructed to classify patients' ultrasound images. It was found that the

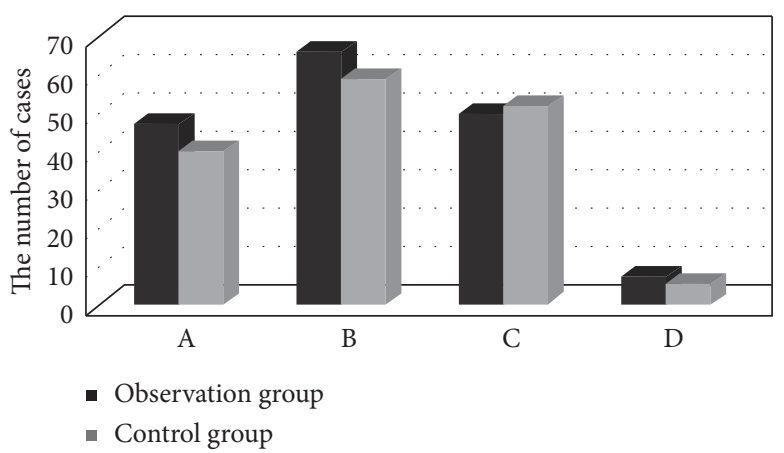

Figure 10: Contrast of natural conception between the two groups. (a) natural conception; (b) cervix not opened; (c) pregnancy was singleton; (d) with history of premature delivery.

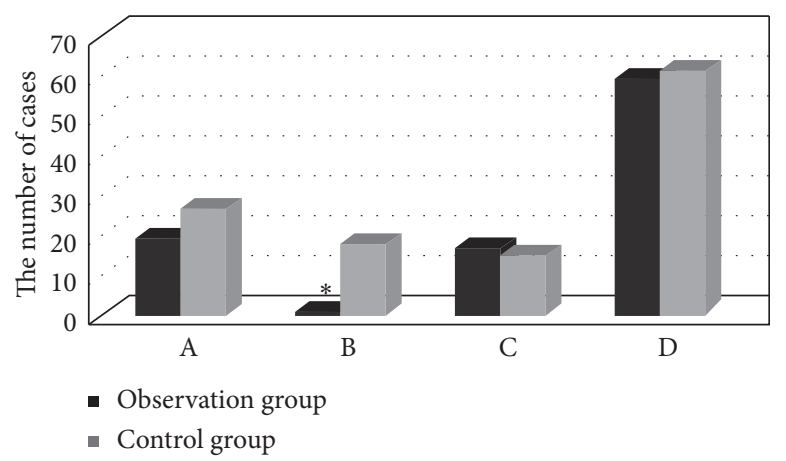

FIGURE 11: Contrast of assisted conception between the two groups ( ${ }^{*}$ in the figure represents $P<0.05$ ): (a) assisted reproduction conception; (b) cervical opening; (c) pregnancy as twins; (d) no history of preterm birth.

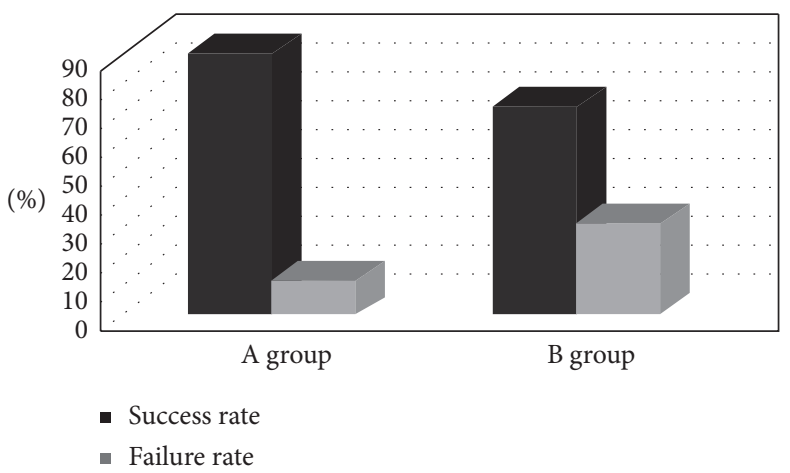

Figure 12: Contrast of surgical success rate of CC before and after 17 weeks in the observation group $\left({ }^{*}\right.$ meant $\left.P<0.05\right)$.

sensitivity of the CNN + LSTM algorithm was $98.27 \%$, the specificity was $91.73 \%$, the accuracy was $97.72 \%$, and the running time was $(10.72 \pm 3.81) s$. Its sensitivity, specificity, and accuracy were evidently greater than SDD300 and manual sorting $(P<0.05)$. The running time of the algorithm was shorter relative to SDD300 and manual sorting $(P<0.05)$. Wang et al. $[13]$ compared the pregnancy outcomes of patients who had not undergone cervical treatment and those who had undergone CC. The pregnancy outcome 


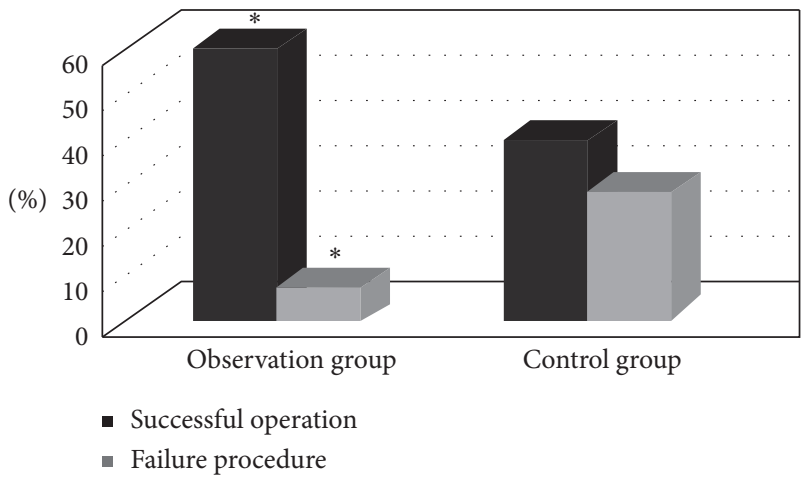

FIgURE 13: Contrast of the surgical success rate between two groups of patients ( ${ }^{*}$ indicated $\left.P<0.05\right)$.

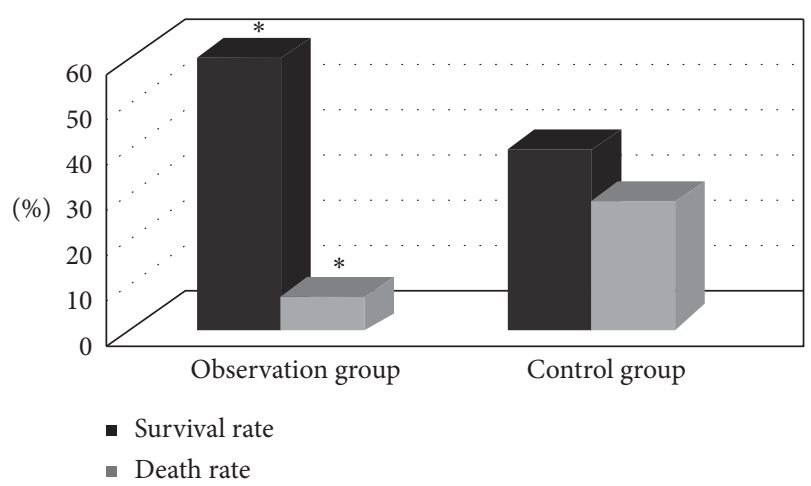

Figure 14: Contrast of neonatal survival rates between the two groups of patients ( ${ }^{*}$ indicated $\left.P<0.05\right)$.

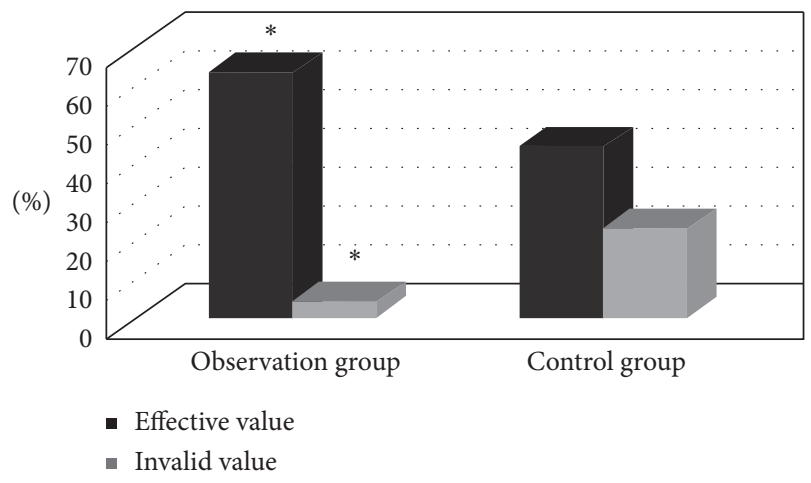

Figure 15: Contrast of clinical effects between the two groups of patients ( ${ }^{*}$ indicated $P<0.05$ ).

of patients treated with CC was better than that of patients without cervical treatment. This was exactly consistent with the results of this study.

In this work, CNN and LSTM model algorithm was employed for CC treatment. Fifty seven cases of neonates survived successfully, accounting for $85.1 \%$. The survival rate of observation group was notably higher relative to controls $(P<0.05)$. The result was consistent with the results reported by $\mathrm{McDermott}$ et al. [14] that CC for CIC was superior to vaginal progesterone in increasing the survival rate of newborns. It suggested that the optimal time to perform CC was in 14-18 weeks of gestational age [15]. Among the patients under investigation, in the observation group, 40 patients in group A were treated with CC before 17 weeks, 36 patients were successfully treated with a success rate of $89.6 \%$, and 4 patients failed with a failure rate of $10.4 \%$; in group B, 27 patients were treated with CC after 17 weeks, 19 patients were successfully treated with a success rate of $70.4 \%$, and 8 patients failed with a failure rate of $29.6 \%$. The observation group had a notably higher surgical success rate versus the control group, which indicated remarkable differences between the two groups $(P<0.05)$. In summary, the observation group had a higher surgical success rate before the $17^{\text {th }}$ week of gestation, and slightly lower surgical success rate after 17 weeks. The results of the treatment were better for cervical ligation. This validated the findings in the study.

\section{Conclusion}

In this work, through the construction of CNN and LSTM algorithms, the ultrasound images of CIC patients in the observation group were analyzed, to study the timing and clinical outcome of CC surgery. The results showed that using CNN and LSTM model algorithms to classify patient ultrasound images can effectively improve diagnosis and treatment efficiency. The surgical success rate, clinical outcome, neonatal survival rate, and clinical effect of observation group were better than controls. Moreover, the optimal surgery time of CC for CIC patients was before 17 weeks of gestation. The disadvantage of this research topic is that the selected patient sample size is small and there is a certain deviation. The scope of application of the research results is also relatively small. In subsequent studies, the sample size of patients will be increased and the scope of the study will be expanded to further explore the clinical treatment of CIC patients. In short, the results provide a certain reference for the clinical treatment of CIC patients.

\section{Data Availability}

The data used to support the findings of this study are available from the corresponding author upon request.

\section{Conflicts of Interest}

The authors declare no conflicts of interest.

\section{Authors' Contributions}

Xuekui Ye and Li Zhang equally to this work.

\section{References}

[1] A. Roman, A. Suhag, and V. Berghella, "Overview of cervical insufficiency: diagnosis, etiologies, and risk factors," Clinical Obstetrics and Gynecology, vol. 59, no. 2, pp. 237-240, 2016, PMID: 27015229.

[2] S. El Gelany, M. H. Mosbeh, E. M. Ibrahim et al., "Placenta Accreta Spectrum (PAS) disorders: incidence, risk factors and outcomes of different management strategies in a tertiary referral hospital in Minia, Egypt: a prospective study," BMC Pregnancy and Childbirth, vol. 19, no. 1, p. 313, 2019. 
[3] S.-W. Wang, L.-L. Ma, S. Huang, L. Liang, and J.-R. Zhang, "Role of cervical cerclage and vaginal progesterone in the treatment of cervical incompetence with/without preterm birth history," Chinese Medical Journal, vol. 129, no. 22, pp. 2670-2675, 2016, PMID: 27823998.

[4] K.-N. Lee, E.-J. Whang, K. H.-J. Chang, J.-E. Song, G.-H. Son, and K.-Y. Lee, "History-indicated cerclage: the association between previous preterm history and cerclage outcome," Obstetrics \& Gynecology Science, vol. 61, no. 1, pp. 23-29, 2018, Epub 2017 Dec 14. PMID: 29372146; PMCID: PMC5780317.

[5] L. M. Negrete and L. B. Spalluto, "Don't be short-sighted: cervical incompetence in a pregnant patient with acute appendicitis," Clinical Imaging, vol. 51, pp. 35-37, 2018, Epub 2018 Jan 31. PMID: 29414522.

[6] S. S. R. Herbst, A. B. Peixoto, E. Araujo Júnior, A. F. Moron, and R. Mattar, "Effects of cervical cerclage on cervical length and the impact of changes in cervical length on pregnancy prognosis," Ceská Gynekologie, vol. 83, no. 5, pp. 341-347, 2018, English. PMID: 30848137.

[7] K. Kosińska-Kaczyńska, I. Szymusik, D. Bomba-Opoń, R. Brawura-Biskupski-Samaha, P. Wegrzyn, and M. Wielgoś, "Effective treatment of cervical incompetence in a monochorionic monoamniotic twin pregnancy with a rescue cervical cerclage and pessary--a case report and review of literature," Ginekologia Polska, vol. 83, no. 12, pp. 946-949, 2012, PMID: 23488300.

[8] K. Seo, S. Dohi, T. Ishikawa, K. Ichizuka, A. Sekizawa, and M. Nagatsuka, "Modified laparoscopic cervicoisthmic cerclage in early pregnancy for refractory cervical incompetence: a case report," Journal of Obstetrics and Gynaecology Research, vol. 45, no. 8, pp. 1597-1602, 2019, Epub 2019 May 28. PMID: 31137082 .

[9] A. S. El-Agwany, "Conservative treatment of placenta previa accreta with cervical isthmic opposition suturing followed by bilateral internal iliac artery ligation," International Journal of Gynecology \& Obstetrics, vol. 135, no. 3, pp. 329-330, 2016, Epub 2016 Aug 30. PMID: 27793334.

[10] A. Sanabria, L. P. Kowalski, and F. Tartaglia, "Inferior thyroid artery ligation increases hypocalcemia after thyroidectomy: a meta-analysis," The Laryngoscope, vol. 128, no. 2, pp. 534-541, 2018, Epub 2017 May 31. PMID: 28561328.

[11] J.-L. Delafontaine, R. Candal, R. Ranganath, R. K. White, and Q. D. Chu, "Cervical esophageal exclusion with temporary suture ligation: a novel technique in the management of esophageal perforations," The American Surgeon, vol. 86, no. 5, pp. 513-515, 2020.

[12] J. Zhang, Z. Zhang, X. Ji et al., "Deep learning convolutional neural network in diagnosis of serous effusion in patients with malignant tumor by tomography," The Journal of Supercomputing, vol. 15, 2021.

[13] C.-Y. Wang, H.-H. Pan, C.-C. Chang, and C.-K. Lin, "Outcomes of hypogastric artery ligation and transcatheter uterine artery embolization in women with postpartum hemorrhage," Taiwanese Journal of Obstetrics \& Gynecology, vol. 58, no. 1, pp. 72-76, 2019.

[14] A. M. McDermott, E. O’Cathain, B. W. Carey, P. O’Sullivan, and P. Sheahan, "Sphenopalatine artery ligation for epistaxis," Otolaryngology-Head and Neck Surgery, vol. 154, no. 3, pp. 547-552, 2016.

[15] G. de Bonnecaze, Y. Gallois, F. Bonneville, S. Vergez, B. Chaput, and E. Serrano, "Transnasal endoscopic sphenopalatine artery ligation compared with embolization for intractable epistaxis: a long-term analysis," American Journal of Rhinology \& Allergy, vol. 32, no. 3, pp. 188-193, 2018. 